\title{
Letramento em saúde de cuidadores domiciliares de uma capital brasileira
}

\author{
Health literacy of home caregivers in a Brazilian capital \\ Alfabetización en salud de cuidadores domiciliarios de una capital brasileña
Thales Antônio Martins Soares ${ }^{1}$ ic https://orcid.org/0000-0001-5780-6476
Virginia Visconde Brasil' 1 io https://orcid.org/0000-0002-0279-9878
Katarinne Lima Moraes² is https://orcid.org/0000-0001-6169-0461
Laidilce Teles Zatta Santos ${ }^{3}$ ib https://orcid.org/0000-0003-0566-6883
Vanessa da Silva Carvalho Vila ${ }^{3}$ il https://orcid.org/0000-0002-1785-8682
Laerte Honorato Borges Júnior ${ }^{4}$ il https://orcid.org/0000-0001-9593-9405

Como citar: LT, Vila VS, Borges Júnior LH. Letramento em saúde de cuidadores domiciliares de uma capital brasileira. Acta Paul Enferm. 2021;34:eAPE002255.

DOI

http://dx.doi.org/10.37689/actaape/2021A0002255

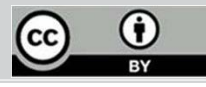

Descritores

Letramento em saúde; Cuidadores; Visita domicilia

Keywords

Health literacy; Caregivers; House calls

Descriptores

Alfabetización en salud; Cuidadores; Visita domiciliaria

\section{Submetido \\ 17 de Agosto de 2020 \\ Aceito \\ 7 de Dezembro de 2020}

Autor correspondente

Thales Antônio Martins Soares E-mail: enf.thalesams@gmail.com

\section{Resumo}

Objetivo: Analisar as condições de letramento em saúde dos cuidadores de usuários vinculados ao Serviço de Atenção Domiciliar de uma capital brasileira.

Métodos: Estudo transversal realizado com 90 cuidadores vinculados ao Serviço de Atenção Domiciliar, de município na região central do Brasil. Coleta realizada no domicílio dos usuários, usando questionário sociodemográfico e a versão brasileira do Health Literacy Questionnaire (HLQ-Br). Utilizada estatística descritiva para variáveis sociodemográficas, a média dos escores nas escalas do HLQ e testes não paramétricos para medidas de associação do letramento em saúde.

Resultados: A maioria dos entrevistados era mulher $(90,0 \%)$, com companheiro $(58,9 \%)$, vivia no mesmo local do usuário $(75,6 \%)$, possuía renda pessoal até um salário mínimo $(72,2 \%)$, não possuía o hábito de ler $(57,8 \%)$, estudou nove anos ou mais $(53,3 \%)$, era cuidador informal $(91,1 \%)$ e tinha pais que não estudaram $(42,2 \%)$. Limitações foram identificadas nas escalas "Cuidado ativo em saúde" e "Navegar no sistema de saúde". As condições de letramento em saúde foram influenciadas negativamente por não ter hábito de ler; escolaridade do cuidador e de seus pais; pelo fato de ser cuidador informal; pela renda e tempo cuidando dos pacientes. As potencialidades foram relacionadas ao acesso a quem entende e apoia 0 cuidador; à compreensão das informações sobre saúde e saber o que fazer, e a entenderem o que os profissionais de saúde thes pedem.

Conclusão: Necessário incorporar os princípios do letramento em saúde no cotidiano dos cuidadores, profissionais e gestores para melhores desfechos e decisões em saúde no contexto da assistência no domicílio.

\section{Abstract}

Objective: To analyze the health literacy conditions of caregivers of Brazilian Home Care Program users.

Methods: Cross-sectional study conducted with 90 caregivers of the Brazilian Home Care Program in a municipality in central Brazil. Data collection was performed at users' homes using a sociodemographic questionnaire and the Brazilian version of the Health Literacy Questionnaire (HLQ-Br). Descriptive statistics were used for sociodemographic variables, the mean scores of the HLQ scales and non-parametric tests for measures of association of health literacy.

Results: Most respondents were women (90.0\%), with a partner (58.9\%), lived in the same location as the user $(75.6 \%)$, had a personal income of up to one minimum wage $(72.2 \%)$, did not have the habit of reading $(57.8 \%)$, studied nine years or more $(53.3 \%)$, were informal caregivers $(91.1 \%)$ and had parents who did not study (42.2\%). Health literacy limitations were identified in the scales "Actively managing my health" and 
"Navigating the healthcare system". Health literacy conditions were negatively influenced by the infrequent reading habit; schooling of caregivers and their parents; the fact of being an informal caregiver; by the low income and less time as a caregiver. The strengths of caregivers were related to the access to those who understand and support the caregiver; understanding health information and knowing what to do, and understanding health professionals' requests.

Conclusion: It is necessary to incorporate the principles of health literacy in the routine of caregivers, professionals and managers for better health outcomes and decisions in the home care context.

\section{Resumen}

Objetivo: Analizar las condiciones de alfabetización en salud de los cuidadores de usuarios vinculados al Servicio de Atención Domiciliaria de una capital brasileña.

Métodos: Estudio transversal realizado con 90 cuidadores vinculados al Servicio de Atención Domiciliaria de un municipio en la región central de Brasil. La recopilación fue realizada en el domicilio de los usuarios, mediante un cuestionario sociodemográfico y la versión brasileña del Health Literacy Questionnaire (HLQ-Br). Se utilizó la estadística descriptiva para variables sociodemográficas, el promedio de puntuaciones de las escalas del HLQ y pruebas no paramétricas para medidas de asociación de la alfabetización en salud.

Resultados: La mayoría de los entrevistados era mujer $(90,0 \%)$, con compañero $(58,9 \%)$, vivía en el mismo lugar que el usuario $(75,6 \%)$, tenía un ingreso personal de hasta un salario mínimo (72,2\%), no tenía el hábito de leer (57,8 \%), estudió nueve años o más (53,3 \%), era cuidador informal (91,1 \%) y sus padres no estudiaron (42,2\%). Se identificaron limitaciones en las escalas "Cuidado activo de la salud" y "Navegar en el sistema de salud". Las condiciones de alfabetización en salud fueron influenciadas de forma negativa por no tener el hábito de leer, por la escolaridad del cuidador y de sus padres, por el hecho de ser cuidador informal y por los ingresos y tiempo cuidando a los pacientes. Las posibilidades se relacionaron con el acceso a quien entiende y apoya al cuidador, con la comprensión de la información sobre salud y saber qué hacer y entender lo que los profesionales de la salud les piden.

Conclusión: Es necesario incorporar los principios de la alfabetización en salud en la cotidianidad de los cuidadores, profesionales y administradores para obtener mejores resultados y tomar mejores decisiones respecto a la salud en el contexto de la atención domiciliaria.

\section{Introdução}

O letramento em saúde é definido pela Organização Mundial de Saúde como as "habilidades cognitiva e social que determinam a motivação e a capacidade dos indivíduos de obter acesso, compreender e utilizar a informação de maneira a promover e manter uma boa saúde". ${ }^{(1)}$ Determinar as condiçôes de letramento em saúde dos indivíduos ajuda a melhorar a qualidade das informaçóes prestadas pelos serviços, a participação da comunidade no autogerenciamento e tomada de decisóes, o planejamento de serviços e a educação em saúde pública. ${ }^{(2)}$

Limitaçóes neste constructo contribuem para maiores índices de mortalidade, aumento da taxa de hospitalização, redução do uso de serviços de prevenção, maiores gastos, dificuldade em navegar no sistema de saúde (localizar, ter acesso e decidir sobre o serviço e profissionais de saúde), comunicação ineficaz com os profissionais e dificuldades na compreensão das orientaçóes fornecidas pelos serviços de saúde. ${ }^{(3,4)}$

Para que as pessoas e serviços possam melhor gerir a saúde, é fundamental avaliar este constructo em diferentes contextos de cuidado. ${ }^{(5,6)}$ Isso inclui os cuidados à saúde prestados em domicílio por cuidadores. Os cuidadores são definidos pelo Ministério da Saúde como pessoas que gastam maior parte do seu tempo na prestação de cuidados, podendo ser ou não membro da família. ${ }^{(7)}$
A literatura tem descrito que o letramento em saúde de cuidadores domiciliares apresenta limitaçôes e fragilidades, o que contribui para falhas no cuidado em domicílio e gera piores resultados em saúde, tais como dificuldade de navegar nos sistemas de saúde ${ }^{(8)}$ e de compreensão da patologia de seus pacientes; ${ }^{(9)}$ negligência no cuidado; ${ }^{(10)}$ dificuldade de entender as orientaçóes fornecidas pelos profissionais e não ser capaz de identificar as limitaçôes do letramento de quem está sendo cuidado. ${ }^{(11)}$

A presença do cuidador domiciliar é fundamental para o funcionamento das açôes de saúde, na modalidade de cuidado que realiza açóes de prevenção, tratamento, reabilitação, paliação e promoção de saúde em domicílio, denominada Serviço de Atenção Domiciliar - SAD, no contexto do sistema público de saúde brasileiro. ${ }^{(12)}$

E assim, é necessário incluir a avaliação do letramento em saúde do cuidador domiciliar como prioridade nas políticas públicas de saúde, objetivando melhorar os resultados de saúde do próprio cuidador e do indivíduo que ele cuida. ${ }^{(13)}$

Para verificar o quanto este indivíduo é capacitado para ser bem-sucedido nos distintos contextos de saúde, indicar se ele compreende orientaçóes dos profissionais e aplica tais deliberaçóes em seu cotidiano e ainda, se consegue navegar no sistema e gerenciar sua saúde garantindo acesso e uso equitativo aos serviços, objetivou-se analisar as condiçóes de letramento em 
saúde dos cuidadores de usuários vinculados ao Serviço de Atenção Domiciliar de uma capital brasileira.

\section{Métodos}

Trata-se de um estudo transversal analítico conduzido com 90 cuidadores vinculados ao Serviço de Atenção Domiciliar que atende usuários do Sistema Único de Saúde, em município da região central do Brasil no ano 2019.

Este serviço funciona de maneira descentralizada e as equipes são distribuídas por regióes na cidade. $\mathrm{O}$ perfil de usuários atendidos pelas equipes multiprofissionais é de indivíduos com patologias crônicas, doenças infectocontagiosas, enfermidades oncológicas, desnutrição e que necessitam de oxigenioterapia domiciliar.

Foram incluídos no estudo todos os cuidadores com 18 anos ou mais e vinculados ao Serviço de Atenção Domiciliar há pelo menos 30 dias - esse tempo foi considerado como período de adaptaçáo às atividades do SAD. Foram excluídos os cuidadores de pacientes que vieram a óbito, que tinham recebido alta do serviço, que estavam internados e aqueles que não foram localizados após três tentativas de contato.

Foi solicitada autorização de uso da versão brasileira do Health Literacy Questionnaire (HLQ) à universidade australiana (e-mail hl-info@swin.edu.au) e aos autores da versão brasileira. Os participantes foram esclarecidos sobre os objetivos do estudo e assinaram Termo de Consentimento Livre e Esclarecido.

A coleta de dados aconteceu no domicílio dos usuários cadastrados no SAD, durante as visitas domiciliares realizadas pelas equipes multidisciplinares, no período de março a junho de 2019.

A equipe de pesquisadores foi treinada para coleta de dados. Foram utilizados a versão brasileira do Health Literacy Questionnaire - HLQ-Br e um questionário para caracterização sociodemográfica. O letramento em saúde foi variável de desfecho.

O HLQ é um instrumento multidimensional que foi traduzido para o português brasileiro em 2018 e validado com 794 brasileiros adultos usuários do SUS de três regiôes distintas do Brasil, sendo denominado HLQ-Br. Obteve boas propriedades psicométricas no processo de validação para o português brasileiro. ${ }^{(14)}$
Contém 44 itens distribuídos em nove escalas que são divididas em duas partes, ${ }^{(15)}$ descritos na figura $1 .^{(14)}$

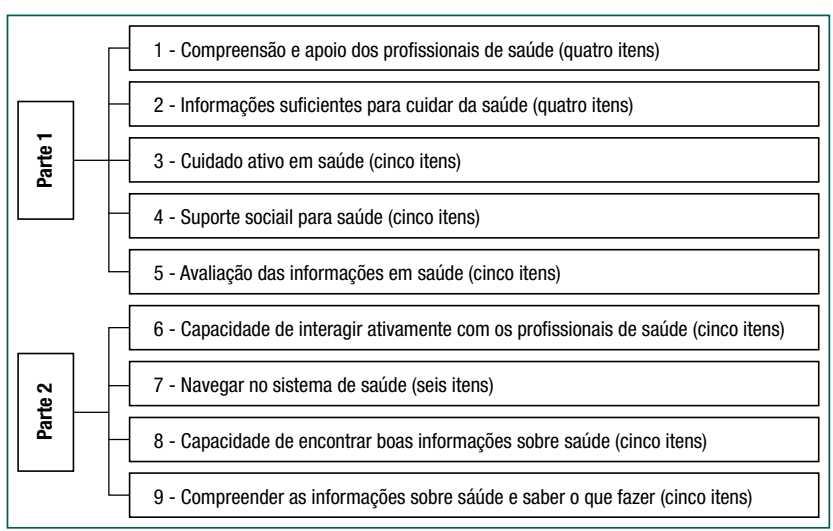

Fonte: Moraes KL, Brasil V, Mialhe FL, Sampaio HA, Sousa AL, Canhestro MR. Validation of the Health Literacy Questionnaire (HLQ) to Brazilian Portuguese (prelo). Acta Paul Enferm. 2020;34(1). ${ }^{(14)}$

Figura 1. Escalas do Health Literacy Questionnaire - versão brasileira

A Parte 1 tem quatro opçóes de respostas entre "discordo totalmente" e "concordo totalmente" (escores 1 - 4); a Parte 2 tem cinco opçôes de respostas entre "não consigo fazer ou sempre difícil" e "sempre fácil" (escores 1-5). Este questionário não fornece pontuação global e sim escores com a média individual das nove escalas. A pontuação mais próxima do limite superior ou inferior indica, respectivamente, as potencialidades e as limitaçóes dos cuidadores no cuidado da própria saúde. ${ }^{(15)}$

O tempo de preenchimento do HLQ varia dependendo das habilidades do entrevistado quando auto administrado ou quando oralmente administrado. ${ }^{(15)}$ Neste estudo, o tempo necessário para aplicação do questionário sociodemográfico foi 3,29 $\pm 1,00$ minutos (variação 1 - 7), mediana 3 minutos. A aplicação do HQL-Br durou em média $12,67 \pm 4,38$ minutos $(6-29)$ e mediana 11 minutos.

Para facilitar a compreensão dos participantes sobre a escala tipo Likert dos itens do HLQ-Br foi criado um recurso visual pelos autores, associando expressóes faciais e cores diferentes para as respostas de concordância/discordância com as afirmaçôes, e de facilidade/dificuldade de realizar açóes (Figura 2).

A normalidade dos dados foi analisada pelo teste de Shapiro-Wilk. Para fazer as comparaçóes dos escores com as variáveis categóricas de dois níveis utilizou-se o teste de Mann Whitney, enquanto que, 


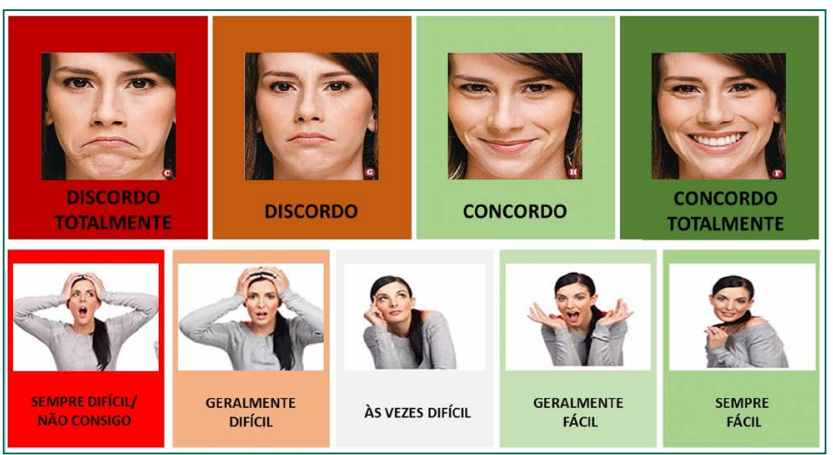

Figura 2. Recurso visual para facilitar a compreensão das respostas do $\mathrm{HLQ}-\mathrm{Br}$

para as com três ou mais níveis foi utilizado o teste de Kruskal-Wallis.

O cálculo dos escores foi realizado pela soma de cada item das escalas e esse valor foi dividido pelo número de itens da escala, sendo o valor apresentado como a média de pontuação.

Foi apresentado o d de Cohen para medida de tamanho de efeito ou a diferença em desvios padróes entre as médias de dois grupos, sendo considerado pequeno, entre 0,20 e 0,50 ; médio, entre 0,50 e 0,80 ; e grande, se superior a $0,80 .{ }^{(16)} \mathrm{O}$ valor de $\mathrm{p}<0,05$ foi assumido para significância estatística.

$\mathrm{O}$ estudo seguiu os preceitos éticos vigentes no Brasil para pesquisa com seres humanos (CAAE 06178519.0.0000.5078).

\section{Resultados}

A maioria dos cuidadores era do sexo feminino (90,0\%); possuía companheiro fixo $(58,89 \%)$; vivia na residência de quem cuidava $(75,56)$; não possuía hábito de leitura $(57,78 \%)$; não exercia atividade laboral remunerada $(80,0 \%)$ e era cuidador informal $(91,11 \%)$; possuía pais com nove anos ou mais de escolaridade (43,33\%). Estudaram principalmente em escola pública $(84,44 \%)$, por nove anos ou mais $(53,33 \%)$. A renda pessoal predominante foi de até um salário mínimo $(72,22 \%)$ e a renda familiar de até dois salários $(52,22 \%)$. Eram cuidadores principalmente os filhos $(34,44 \%)$, há aproximadamente entre seis meses e cinco anos (45,56\%).

Por meio análise descritiva e das médias de cada item das escalas do questionário HQL-Br, foram identificadas limitaçóes no letramento em saúde em termos do Cuidado ativo e informaçóes suficientes para cuidar da saúde (parte 1); e Navegar no sistema de saúde (parte 2). As médias mais elevadas representativas das potencialidades do letramento em saúde foram Suporte social e Avaliação das informaçóes em saúde (parte 1) e Capacidade de interagir ativamente com profissionais de saúde, como descrito na tabela 1.

Tabela 1. Escores médios das escalas e dos itens do Health Literacy Questionnaire-versão brasileira que apresentaram maiores limitações e potencialidades do letramento em saúde de 90 cuidadores vinculados ao Serviço de Atenção Domiciliar

\begin{tabular}{|c|c|}
\hline Escalas e Questões do HLQ-Br & Média (DP) \\
\hline \multicolumn{2}{|l|}{ Parte 1 (Escores 1-4) } \\
\hline 1 - Compreensão e apoio dos profissionais de saúde & $2,69(0,55)$ \\
\hline Q22 - Eu posso contar com pelo menos um profissional da saúde & $2,81(0,58)$ \\
\hline $\begin{array}{l}\text { Q17 - Eu tenho os profissionais de saúde que necessito para me ajudar a } \\
\text { decidir o que eu preciso fazer }\end{array}$ & $2,53(0,66)$ \\
\hline 2 - Informações suficientes para cuidar da saúde & $2,50(0,47)$ \\
\hline Q01 - Na minha opinião, tenho boas informações sobre saúde & $2,63(0,63)$ \\
\hline $\begin{array}{l}\text { Q10 - Eu tenho informação suficiente para me ajudar a lidar com meus } \\
\text { problemas de saúde }\end{array}$ & $2,42(0,75)$ \\
\hline 3 - Cuidado ativo da saúde & $2,46(0,49)$ \\
\hline Q18 - Eu decido meus próprios objetivos sobre saúde e boa forma & $2,76(0,66)$ \\
\hline Q06 - Eu gasto bastante tempo envolvido com minha saúde & $2,01(0,77)$ \\
\hline 4 - Suporte social para saúde & $2,72(0,53)$ \\
\hline Q03 - Eu tenho acesso a várias pessoas que me entendem e me apoiam & $2,96(0,70)$ \\
\hline $\begin{array}{l}\text { Q15 - Eu tenho pelo menos uma pessoa que pode me acompanhar às } \\
\text { consultas médicas }\end{array}$ & $2,44(0,84)$ \\
\hline 5 - Avaliação das informações em saúde & $2,72(0,49)$ \\
\hline Q04 - Eu comparo informações de saúde obtidas de fontes diferentes & $2,77(0,70)$ \\
\hline $\begin{array}{l}\text { Q12 - Eu sempre comparo informações de saúde obtidas de fontes } \\
\text { diferentes e decido o que é melhor para mim }\end{array}$ & $2,67(0,67)$ \\
\hline \multicolumn{2}{|l|}{ Parte 2 (Escores 1-5) } \\
\hline 6 - Capacidade de interagir ativamente com profissionais de saúde & $3,50(0,80)$ \\
\hline $\begin{array}{l}\text { Q20 - Fazer perguntas aos profissionais de saúde para conseguir as } \\
\text { informações que você precisa }\end{array}$ & $3,86(1,07)$ \\
\hline $\begin{array}{l}\text { Q02 - Ter certeza que os profissionais de saúde entendem os seus } \\
\text { problemas corretamente }\end{array}$ & $3,22(1,22)$ \\
\hline 7 - Navegar no sistema de saúde & $2,84(0,89)$ \\
\hline Q11 - Decidir qual profissional de saúde você precisa consultar & $3,31(1,35)$ \\
\hline Q01 - Encontrar o serviço de saúde adequado & $2,40(1,21)$ \\
\hline 8 - Capacidade de encontrar boas informações sobre saúde & $3,14(0,88)$ \\
\hline $\begin{array}{l}\text { Q14 - Conseguir informações sobre saúde em linguagem que você } \\
\text { entenda }\end{array}$ & $3,28(1,29)$ \\
\hline Q18 - Conseguir informações sobre saúde sozinho & $2,98(1,32)$ \\
\hline 9 - Compreender as informações sobre saúde e saber o que fazer & $3,39(0,81)$ \\
\hline $\begin{array}{l}\text { Q21 - Entender o que os profissionais de saúde estão pedindo que você } \\
\text { faça }\end{array}$ & $3,88(1,17)$ \\
\hline Q17 - Ler e entender todas as informações em rótulos de medicamentos & $2,92(1,33)$ \\
\hline
\end{tabular}

Q - Questão; DP - Desvio Padrão

As tabelas 2 e 3 mostram os padróes dos escores do HLQ de acordo com o status sociodemográfico. Estas descriçóes consideraram, especialmente, as diferenças significativas observadas nas médias e respectivas escalas do HLQ-Br e o tamanho do efeito médio e grande. A análise da associação e o tama- 
Tabela 2. Diferenças evidenciadas na análise da associação entre os escores médios das escalas 1 a 5 do questionário Health Literacy Questionnaire - versão brasileira, com as variáveis sociodemográficas de 90 cuidadores vinculados ao Serviço de Atenção Domiciliar

\begin{tabular}{|c|c|c|c|c|c|c|c|}
\hline \multirow{2}{*}{ Variáveis (n) } & & \multicolumn{2}{|c|}{ Escala 1} & \multicolumn{2}{|c|}{ Escala 2} & \multicolumn{2}{|c|}{ Escala 5} \\
\hline & & Média (E.P.) & $d^{1}$ & Média (E.P.) & $d^{1}$ & Média (E.P.) & $d^{1}$ \\
\hline \multirow{2}{*}{ Tipo de cuidador } & Formal (8) & $3,00(0,09)$ & - & $2,69(0,1)$ & - & $3,13(0,12)$ & - \\
\hline & Informal (82) & $2,66(0,06)$ & 0,62 & $2,48(0,05)$ & 0,44 & $2,68(0,05)$ & 0,93 \\
\hline$p$-value ${ }^{2}$ & & 0,055 & & 0,154 & & 0,008 & \\
\hline \multirow[t]{2}{*}{ Hábito de leitura } & Não (52) & $2,59(0,08)$ & - & $2,42(0,07)$ & - & $2,61(0,07)$ & - \\
\hline & $\operatorname{Sim}(38)$ & $2,83(0,07)$ & 0,45 & $2,61(0,07)$ & 0,41 & $2,87(0,08)$ & 0,56 \\
\hline$p$-value ${ }^{2}$ & & 0,030 & & 0,077 & & 0,004 & \\
\hline \multirow[t]{2}{*}{ Escolaridade cuidador } & $\leq 9$ anos (42) & $2,59(0,09)$ & - & $2,43(0,07)$ & - & 2,48 $(0,07)$ & - \\
\hline & $>9$ anos (48) & $2,78(0,08)$ & 0,34 & $2,57(0,07)$ & 0,30 & $2,93(0,06)$ & 1,02 \\
\hline$p$-value ${ }^{2}$ & & 0,051 & & 0,247 & & $<0,001$ & \\
\hline \multirow[t]{2}{*}{ Renda pessoal (SM) } & $\leq 1(65)$ & $2,63(0,07)$ & - & $2,46(0,05)$ & - & $2,64(0,06)$ & - \\
\hline & $>1(25)$ & $2,83(0,09)$ & 0,36 & $2,62(0,1)$ & 0,35 & $2,94(0,08)$ & 0,63 \\
\hline$p$-value ${ }^{2}$ & & 0,102 & & 0,193 & & 0,006 & \\
\hline \multirow[t]{2}{*}{ Renda familiar (SM) } & $\leq 2(47)$ & $2,56(0,08)$ & - & $2,39(0,06)$ & - & $2,63(0,07)$ & - \\
\hline & $>2(43)$ & $2,83(0,08)$ & 0,51 & $2,62(0,07)$ & 0,50 & $2,82(0,07)$ & 0,39 \\
\hline$p$-value ${ }^{2}$ & & 0,022 & & 0,037 & & 0,114 & \\
\hline \multirow[t]{3}{*}{ Escolaridade pais (ano) } & Nenhuma (33) & $2,67(0,09)$ & - & $2,47(0,07)$ & - & $2,58(0,07)$ & - \\
\hline & $\leq 9$ anos (13) & $2,46(0,14)$ & 0,39 & $2,37(0,11)$ & 0,25 & $2,43(0,14)$ & 0,33 \\
\hline & $>9$ anos (39) & $2,78(0,09)$ & 0,20 & $2,58(0,08)$ & 0,22 & $2,95(0,07)$ & 0,85 \\
\hline$p$-value ${ }^{3}$ & & 0,098 & & 0,344 & & $<0,001$ & \\
\hline \multirow[t]{3}{*}{ Tempo como cuidador } & $<6$ meses (18) & $2,47(0,14)$ & - & $2,35(0,1)$ & - & $2,62(0,12)$ & - \\
\hline & 6 meses $<5$ anos (41) & $2,84(0,08)$ & 0,70 & $2,62(0,07)$ & 0,60 & $2,86(0,08)$ & 0,48 \\
\hline & $>5$ anos (31) & $2,62(0,1)$ & 0,26 & $2,44(0,08)$ & 0,21 & $2,59(0,08)$ & 0,06 \\
\hline$p$-value ${ }^{3}$ & & 0,056 & & 0,073 & & 0,031 & \\
\hline \multirow[t]{4}{*}{ Tipo escola } & Pública (76) & $2,65(0,06)$ & - & $2,52(0,05)$ & - & $2,73(0,05)$ & - \\
\hline & Privada (5) & $3,15(0,13)$ & 0,92 & $2,55(0,17)$ & 0,08 & $2,84(0,23)$ & 0,27 \\
\hline & Ambas (5) & $3,05(0,05)$ & 0,74 & $2,75(0,3)$ & 0,51 & $3,2(0,33)$ & 1,09 \\
\hline & Nenhuma (4) & $2,31(0,4)$ & 0,61 & $1,88(0,22)$ & 1,46 & $1,8(0,29)$ & 2,23 \\
\hline$p$-value ${ }^{3}$ & & 0,029 & & 0,056 & & 0,007 & \\
\hline
\end{tabular}

1d de Cohen; ${ }^{2}$ Mann Whitney; 3 Kruskal-Wallis; Negrito: valores significativos e tamanhos de efeito grande; SM: salário mínimo; E.P: Erro Padrão.

Tabela 3. Diferenças evidenciadas na análise da associação entre os escores médios das escalas 6 a 9 do questionário Health Literacy Questionnaire - versão brasileira, com as variáveis sociodemográficas de 90 cuidadores vinculados ao Serviço de Atenção Domiciliar

\begin{tabular}{|c|c|c|c|c|c|c|c|c|c|}
\hline \multirow{2}{*}{ Variáveis (n) } & & \multicolumn{2}{|c|}{ Escala 6} & \multicolumn{2}{|c|}{ Escala 7} & \multicolumn{2}{|c|}{ Escala 8} & \multicolumn{2}{|c|}{ Escala 9} \\
\hline & & Média (E.P.) & $d^{1}$ & Média (E.P.) & $d^{1}$ & Média (E.P.) & $d^{1}$ & Média (E.P.) & $d^{1}$ \\
\hline \multirow[t]{2}{*}{ Tipo de cuidador } & Formal (8) & $3,58(0,23)$ & - & $2,81(0,29)$ & - & $3,75(0,26)$ & - & $3,38(0,17)$ & - \\
\hline & Informal (82) & $3,5(0,09)$ & 0,10 & $2,84(0,10)$ & 0,03 & $3,08(0,10)$ & 0,78 & $3,39(0,09)$ & 0,02 \\
\hline$p$-value ${ }^{2}$ & & 0,870 & & 0,809 & & 0,035 & & 0,966 & \\
\hline \multirow[t]{2}{*}{ Hábito de leitura } & Não (52) & $3,35(0,12)$ & - & $2,65(0,12)$ & - & $2,86(0,11)$ & - & $3,14(0,11)$ & - \\
\hline & Sim (38) & $3,72(0,11)$ & 0,48 & $3,1(0,14)$ & 0,53 & $3,52(0,14)$ & 0,80 & $3,72(0,12)$ & 0,76 \\
\hline$p$-value ${ }^{2}$ & & 0,056 & & 0,014 & & 0,001 & & $<0,001$ & \\
\hline \multirow[t]{2}{*}{ Moram com os pacientes e renda familiar > um salário } & Não (23) & $3,31(0,14)$ & - & $2,49(0,13)$ & - & $3,03(0,17)$ & - & $3,16(0,15)$ & - \\
\hline & $\operatorname{Sim}(67)$ & $3,57(0,1)$ & 0,32 & $2,96(0,11)$ & 0,54 & $3,17(0,11)$ & 0,15 & $3,47(0,1)$ & 0,38 \\
\hline$p$-value ${ }^{2}$ & & 0,135 & & 0,023 & & 0,597 & & 0,147 & \\
\hline \multirow[t]{2}{*}{ Escolaridade do cuidador } & $\leq 9$ anos (42) & $3,45(0,14)$ & - & $2,57(0,13)$ & - & $2,73(0,12)$ & - & $2,93(0,11)$ & - \\
\hline & $>9$ anos (48) & $3,55(0,1)$ & 0,12 & $3,07(0,13)$ & 0,59 & $3,49(0,12)$ & 0,96 & $3,78(0,1)$ & 1,23 \\
\hline$p$-value ${ }^{2}$ & & 0,877 & & 0,004 & & $<0,001$ & & $<0,001$ & \\
\hline \multirow[t]{2}{*}{ Renda familiar (SM) } & $\leq 2(47)$ & $3,46(0,12)$ & - & $2,69(0,11)$ & - & $2,94(0,13)$ & - & $3,25(0,12)$ & - \\
\hline & $>2(43)$ & $3,56(0,12)$ & 0,13 & $3(0,15)$ & 0,35 & $3,35(0,12)$ & 0,47 & $3,53(0,12)$ & 0,35 \\
\hline$p$-value ${ }^{2}$ & & 0,509 & & 0,081 & & 0,020 & & 0,135 & \\
\hline \multirow[t]{3}{*}{ Escolaridade pais do cuidador } & Sem escolaridade (38) & $3,52(0,15)$ & - & $2,75(0,15)$ & - & $2,82(0,14)$ & - & $3,11(0,13)$ & - \\
\hline & $\leq 9$ anos (13) & $3,08(0,17)$ & 0,53 & $2,27(0,12)$ & 0,59 & $2,72(0,09)$ & 0,13 & $3(0,14)$ & 0,14 \\
\hline & $>9$ anos (39) & $3,63(0,11)$ & 0,14 & $3,12(0,14)$ & 0,42 & $3,58(0,13)$ & 0,89 & $3,79(0,12)$ & 0,89 \\
\hline$p$-value ${ }^{3}$ & & 0,064 & & 0,003 & & $<0,001$ & & $<0,001$ & \\
\hline \multirow[t]{4}{*}{ Tipo de escola } & Pública (76) & $3,47(0,09)$ & - & $2,77(0,10)$ & - & $3,12(0,09)$ & - & $3,37(0,09)$ & - \\
\hline & Privada (5) & $3,72(0,4)$ & 0,31 & $3,47(0,47)$ & 0,81 & $3,56(0,43)$ & 0,53 & $4,04(0,39)$ & 0,85 \\
\hline & Ambas (5) & $3,84(0,38)$ & 0,46 & $3,67(0,37)$ & 1,06 & $4,04(0,23)$ & 1,14 & $3,8(0,34)$ & 0,55 \\
\hline & Não frequentou (4) & $3,4(0,42)$ & 0,09 & $2,34(0,43)$ & 0,51 & $1,75(0,36)$ & 1,68 & $2,3(0,10)$ & 1,41 \\
\hline$p$-value $e^{3}$ & & 0,610 & & 0,077 & & 0,003 & & 0,010 & \\
\hline
\end{tabular}


nho de efeito entre os escores médios das escalas 1 a 5 (Parte 1) do HLQ-Br estão descritas na tabela 2 e, entre os escores médios das escalas 6 a 9 (Parte 2), estão na tabela 3 .

Identificou-se diferenças das médias do HLQ considerando subgrupos em termos do tipo de cuidador, hábito de leitura, escolaridade do cuidador e dos pais, renda pessoal e familiar, tempo do cuidador.

As limitações no letramento em saúde identificadas para a "Compreensão e apoio dos profissionais de saúde (Parte 1)" foram evidenciadas em cuidadores informais, com renda familiar menor que dois salários mínimos, que não tinham hábito de leitura, com escolaridade inferior a nove anos e que nunca frequentaram algum tipo de escola.

Em termos das "Informaçóes suficientes para cuidar da saúde (Parte 1)", as limitaçôes foram entre os cuidadores com renda familiar inferior a dois salários mínimos e que não estudaram. Quanto à "Avaliação das informaçôes em saúde (Parte 1)" foram observados escores inferiores entre os cuidadores informais; que não tinham hábito de leitura; escolaridade do cuidador e dos pais inferior ou igual a nove anos; renda pessoal menor ou igual a um salário mínimo, que cuidavam de seus paciente a mais de cinco anos e que nunca frequentaram nenhum tipo de escola.

A respeito de conseguir "Interagir ativamente com profissionais de saúde" (Parte 2), observou-se limitaçôes nos cuidadores que não possuíam hábito de leitura. Já em relação a "Navegar no sistema de saúde" (Parte 2), as menores médias foram associadas a cuidadores e seus pais com escolaridade inferior ou igual a nove anos e que não possuíam hábito de ler.

Quanto à "Capacidade de encontrar boas informaçôes sobre saúde" (Parte 2), as médias inferiores relacionam-se a ser cuidador informal, não ter hábito de leitura, renda familiar menor ou igual a dois salários mínimos, escolaridade dos pais e cuidador inferior ou igual a 9 anos e não ter frequentado escola. O letramento em termos da "Compreensão das informaçôes sobre saúde e saber o que fazer" (Parte 2) teve limitação para quem não tem o hábito de leitura, escolaridade do cuidador e pais inferior a nove anos e cuidadores que não frequentaram qualquer tipo de escola.

\section{Discussão}

No conhecimento dos autores, este é o primeiro estudo realizado no mundo que analisou as condiçóes de letramento em saúde de cuidadores domiciliares usando um instrumento multidimensional de avaliação. Além de permitir mapear as necessidades em letramento em saúde para indivíduos ou grupo de indivíduos, ${ }^{(17)}$ o HLQ-Br mostrou ser instrumento altamente confiável, com propriedades psicométricas adequadas, assim como ocorreu em outros países onde foi validado. ${ }^{(18,19)}$

Reconhecendo o papel essencial dos cuidadores informais na saúde e no desfecho do cuidado de seus pacientes, o presente estudo mostrou desempenho pouco adequado nas respostas em todas as escalas do questionário aplicado. O limitado letramento em saúde afeta a execução adequada de cuidados, bem como os desfechos de saúde dos beneficiados. ${ }^{(11)}$

A prática do autocuidado entre cuidadores também constitui grande problema. É comum o cuidador se envolver intensamente no cuidar, acarretando o esquecimento de suas próprias necessidades em saúde, com impacto negativo em seu estado físico, psicológico e financeiro. ${ }^{(20)}$

A falta de orientação e o suporte social expóem o cuidador a grande carga de estresse e sobrecarga que pode afetar sua saúde, seu bem estar e sua qualidade de vida. ${ }^{(20)}$ Confirmando registro na literatura sobre cuidadores, o pouco tempo destinado ao cuidado da própria saúde indica que os participantes do estudo podem estar sofrendo sobrecargas físicas e emocionais, pela alta demanda de tempo para cuidar do outro. ${ }^{(21,22)}$

Por outro lado, constituiu fator positivo nesse grupo de cuidadores o suporte social referido, e também a capacidade de compreender as informaçôes em saúde e entender o que os profissionais de saúde pedem. A interação com os profissionais pode favorecer a compreensão. Um estudo apontou que a capacidade de encontrar, entender e usar as informaçóes de saúde é habilidade importante para os 
cuidadores, ${ }^{(23)}$ pois também precisam buscar, acessar e entender informaçóes de saúde em nome dos pacientes de quem cuidam. ${ }^{(24)}$

Esse resultado positivo difere do estudo que menciona a falta de comunicação com os profissionais de saúde como resultado da dificuldade do cuidador em entender a linguagem médica e se sentir sobrecarregado com a quantidade e o tipo de informação fornecida. ${ }^{(25)}$

São relatados problemas de comunicação entre cuidadores e profissionais relacionados à falta de privacidade com os profissionais de saúde durante as conversas e a falta de atenção ao bem-estar dos cuidadores; ${ }^{(26)}$ falta de informaçôes precisas dos profissionais; ${ }^{(25)}$ e ainda, muitos relatam insegurança decorrente da má comunicação no que diz respeito aos cuidados ofertados. ${ }^{(27)} \mathrm{E}$ assim, percebe-se que a comunicação é barreira psicossocial ao cuidado, com mudanças comportamentais necessárias para navegar no sistema de saúde, identificar e alcançar objetivos de saúde.

Os profissionais de saúde devem facilitar o processo de comunicação, gerenciamento e tomada de decisões na saúde de populaçóes. A literatura indica que, embora os cuidadores tenham relatado que os profissionais de saúde são a fonte preferida de informação, ${ }^{(28)}$ outros autores mostram que os cuidadores enfrentam desafios para acessar informaçóes de saúde em ambientes de saúde, pois sentem a necessidade de expressar seus anseios sem a presença do paciente e não recebem a devida importância por parte dos profissionais durante consultas, ${ }^{(29)}$ além da falta de reconhecimento da função de cuidar. ${ }^{(30)}$

A variável escolaridade dos cuidadores e de seus pais pode ser entendida como potencial limitação ou fortaleza no gerenciamento da saúde e desfechos do letramento em saúde. A maior escolaridade dos cuidadores entrevistados influenciou positivamente no desempenho dos escores, assim como em outros estudos, ${ }^{(13,31)}$ reafirmando que pode aprimorar a capacidade de resposta das pessoas frente a seus problemas de saúde. ${ }^{(32)}$

Como não é possível intervir de imediato na escolaridade das pessoas, a reorientação das estratégias públicas eficazes para otimizar os resultados de saúde deve envolver esforço combinado de aperfeiçoar a comunicação entre os profissionais, o sistema de saúde e seus usuários ${ }^{(33)}$ e disponibilizar informaçôes sobre saúde, preparando antecipadamente os cuidadores para assumirem funçóes diretas de cuidar do outro em saúde. ${ }^{(34)}$

A menor renda pessoal e familiar dos cuidadores entrevistados contribuiu para limitaçôes no letramento em saúde. Outros estudos também corroboram estes resultados, confirmando que baixa renda leva a maiores dificuldades para receber informaçôes de saúde e dificuldade de acessar os serviços. ${ }^{(35)}$

Não foram encontrados estudos que tenham analisado a variável leitura associada ao letramento em saúde. O hábito de ler foi avaliado para verificar se compreendiam as informaçóes em saúde e a partir disso, tomar decisôes assertivas, lembrando que o conceito de letramento em saúde não se restringe apenas às habilidades funcionais básicas, como a leitura. É importante ser capaz não apenas ler, mas entender as informaçôes para melhor envolvimento em seus cuidados de saúde. ${ }^{(36)}$

\section{Conclusão}

A renda, escolaridade e hábito de leitura dos cuidadores influenciaram a maioria das escalas do HLQ$\mathrm{Br}$. Os resultados indicam que as condições de letramento em saúde investigadas foram limitadas tanto pela escolaridade dos cuidadores quanto de seus pais e ainda, pela renda mais baixa, pelo hábito pouco frequente de leitura, pelo fato de ser cuidador informal e há menor tempo como cuidador. Mas as condiçóes de letramento em saúde foram favorecidas pelo acesso dos cuidadores a pessoas que os entendem e apoiam, e também ao fato de entenderem o que os profissionais de saúde lhes pedem, compreenderem as informaçóes sobre saúde e saberem o que fazer. Enquanto política pública, a proposta de que o cuidado é melhor em casa deve garantir o acesso e a continuidade do cuidado, pois é responsabilidade dos serviços, mesmo ocorrendo no domicílio. O letramento em saúde é produto da capacidade dos indivíduos, mas também do investimento dos sistemas públicos de saúde nas maneiras de aprender e ensinar em saúde, para aperfeiçoar a prestação de serviços e o 
consequente desfecho. As necessidades em saúde exigem, por direito, a escuta qualificada, a compreensão, o acesso à solução dos problemas identificados e ainda, vínculos de referência e confiança nos integrantes da equipe de saúde. Assim, o usuário será partícipe do processo de cuidar de si próprio e do outro. É preciso ir além da capacidade de leitura e compreensão textual, entendendo o letramento em saúde como a capacidade para agir.

\section{Agradecimentos}

À Coordenação de Aperfeiçoamento de Pessoal de Nível Superior (CAPES; bolsa de mestrado para Thales Antônio Martins Soares) pelo apoio financeiro para esta pesquisa.

\section{Colaborações}

Soares TAM, Brasil VV, Moraes KL, Santos LTZ, Vila VSC e Borges-Júnior LH colaboraram com a concepção do projeto, análise e interpretação dos dados, redação do artigo, revisão crítica relevante do conteúdo intelectual e aprovação final da versão a ser publicada.

\section{Referências}

1. World Health Organization (WHO); Division of Health Promotion, Education and Communications, Health Education and Health Promotion Unit. Health promotion glossary. Geneva: WHO; 1988.

2. Batterham RW, Hawkins M, Collins PA, Buchbinder R, Osborne RH. Health literacy: applying current concepts to improve health services and reduce health inequalities. Public Health. 2016;132(1):3-12.

3. Berkman ND, Sheridan SL, Donahue KE, Halpern DJ, Crotty K. Low health literacy and health outcomes: an updated systematic review. Ann Intern Med. 2011;155(2):97-107.

4. Cajita MI, Cajita TR, Han HR. Health literacy and heart failure: a systematic review. J Cardiovasc Nurs. 2016;31(2):121-30.

5. Institute of Medicine (IOM). Health literacy: improving health, health systems, and health policy around the world: workshop summary. Washington: National Academy of Sciences; 2013.

6. Jessup RL, Osborne RH, Beauchamp A, Bourne A, Buchbinder R. Health literacy of recently hospitalised patients: a cross-sectional survey using the Health Literacy Questionnaire (HLQ). BMC Health Serv Res. 2017 Jan 19;17(1):52.
7. Brasil. Ministério da Saúde. Secretaria de Atenção à Saúde, Departamento de Atenção Básica. Melhor em Casa. A segurança do hospital no conforto do seu lar. Brasília (DF): Ministério da Saúde; 2012.

8. Fields B, Rodakowski J, James AE, Beach S. Caregiver health literacy predicting healthcare communication and system navigation difficulty. Fam Syst Health. 2018;36(4):482-92.

9. Creary S, Adan I, Stanek J, O'Brien SH, Chisolm DJ, Jeffries T, et al. Sickle cell trait knowledge and health literacy in caregivers who receive in-person sickle cell trait education. Mol Genet Genomic Med. 2017;5(6):692-9.

10. Metin S, Demirci H, Metin AT. Effect of health literacy of caregivers on survival rates of patients under palliative care. Scand J Caring Sci. 2019;33(3):669-76.

11. Yuen EYN, Knight T, Ricciardelli LA, Burney S. Health literacy of caregivers of adult care recipients: A systematic scoping review. Health Soc Care Community. 2018;26(2):e191-e206.

12. Brasil. Ministério da Saúde. Atenção Domiciliar. Portaria № 825, de 25 de Abril de 2016. Redefine a Atenção Domiciliar no âmbito do Sistema Único de Saúde (SUS) e atualiza as equipes habilitadas. Brasília(DF): Ministério da Saúde; 2016.

13. Bonaccorsi G, Pieralli F, Innocenti M, Milani C, Del Riccio M, Donzellini $\mathrm{M}$, et al. Health literacy among non-familial caregivers of older adults: a study conducted in Tuscany (Italy). Int J Environ Res Public Health. 2019;16(19):1-12.

14. Moraes KL, Brasil W, Mialhe FL, Sampaio HA, Sousa AL, Canhestro MR. Validation of the Health Literacy Questionnaire ( $\mathrm{HLQ})$ to Brazilian Portuguese (prelo). Acta Paul Enferm. 2020;34(1).

15. Osborne RH, Batterham RW, Elsworth GR, Hawkins M, Buchbinder R. The grounded psychometric development and initial validation of the Health Literacy Questionnaire (HLQ). BMC Public Health. 2013;13(658):658.

16. Cohen J. Statistical power analysis for the behavioral sciences. 2nd ed. Hillsdale (New Jersey): Lawrence Erlbaum Associates; 1998. 680p.

17. Beauchamp A, Batterham RW, Dodson S, Astbury B, Elsworth GR, McPhee $C$, et al. Systematic development and implementation of interventions to optimise health literacy and access (Ophelia). BMC Public Health. 2017;17(1):230.

18. Nolte S, Osborne RH, Dwinger S, Elsworth GR, Conrad ML, Rose M, et al. German translation, cultural adaptation, and validation of the Health Literacy Questionnaire (HLQ). PLoS One. 2017;12(2):e0172340.

19. Kolarcik P, Cepova E, Madarasova Geckova A, Elsworth GR, Batterham RW, Osborne RH. Structural properties and psychometric improvements of the Health Literacy Questionnaire in a Slovak population. Int J Public Health. 2017;62(5):591-604.

20. Jesus IT, Orlandi AA, Zazzett MS. Sobrecarga, perfil e cuidado: cuidadores de idosos em vulnerabilidade social. Rev Bras Geriatr Gerontol. 2018;21(2):199-209.

21. Wittenberg E, Goldsmith J, Ferrell B, Ragan SL. Promoting improved family caregiver health literacy: evaluation of caregiver communication resources. Psychooncology. 2017;26(7):935-42.

22. Cruz MÉ, Silva DV, Carmo JR, Araújo GD, Camisasca LR, Pereira FA, et al. Burden of caregivers of patients under home care. Rev Enferm UFPE On line. 2020;14: e244235

23. Costa TF, Costa KN, Martins KP, Fernandes MG, Brito SS. Sobrecarga de cuidadores familiares de idosos com acidente vascular encefálico. Esc Anna Nery. 2015;19(2):350-5.

24. Smith PD, Martin B, Chewning B, Hafez S, Leege E, Renken J, et al. Improving health care communication for caregivers: A pilot study. Gerontol Geriatr Educ. 2018;39(4):433-44. 
25. Robinson J, Gott M, Ingleton C. Patient and family experiences of palliative care in hospital: what do we know? An integrative review. Palliat Med. 2014;28(1):18-33.

26. Lund L, Ross L, Petersen MA, Groenvold M. The interaction between informal cancer caregivers and health care professionals: a survey of caregivers' experiences of problems and unmet needs. Support Care Cancer. 2015;23(6):1719-33.

27. Caswell G, Pollock K, Harwood R, Porock D. Communication between family carers and health professionals about end-of-life care for older people in the acute hospital setting: a qualitative study. BMC Palliat Care. 2015;14(35):35.

28. Tunin R, Uziely B, Woloski-Wruble AC. First degree relatives of women with breast cancer: who's providing information and support and who'd they prefer. Psychooncology. 2010;19(4):423-30.

29. Swetenham K, Tieman J, Butow P, Currow D. Communication differences when patients and caregivers are seen separately or together. Int J Palliat Nurs. 2015;21(11):557-63.

30. Williams AL, Bakitas M. Cancer family caregivers: a new direction for interventions. J Palliat Med. 2012;15(7):775-83.
31. de Almeida KM, Toye C, Silveira LV, Slatyer S, Hill K, Jacinto AF. Assessment of functional health literacy in Brazilian carers of older people. Dement Neuropsychol. 2019;13(2):180-6.

32. Dodson S, Osicka T, Huang L, McMahon LP, Roberts MA. Multifaceted assessment of health literacy in people receiving dialysis: associations with psychological stress and quality of life. J Health Commun. 2016;21 Supl 2:91-8.

33. Passamai MP, Sampaio HA, Dias AM, Cabral LA. Letramento funcional em saúde: reflexões e conceitos sobre seu impacto na interação entre usuários, profissionais e sistema de saúde. Interface Comun Saude Educ. 2012;16(41):301-14

34. Jiang Y, Sereika SM, Lingler JH, Tamres LK, Erlen JA. Health literacy and its correlates in informal caregivers of adults with memory loss. Geriatr Nurs. 2018;39(3):285-91.

35. Campos AA, Neves FS, Saldanha RF, Duque KC, Guerra MR, Leite IC, et al. Fatores associados ao letramento funcional em saúde de mulheres atendidas pela Estratégia de Saúde da Família. Cad Saude Colet. 2020;28(1):66-76.

36. Jindal P, MacDermid JC. Assessing reading levels of health information: uses and limitations of flesch formula. Educ Health (Abingdon). 2017;30(1):84-8. 